\title{
Digital health technology and mobile devices for the management of diabetes mellitus: state of the art
}

\author{
Rongzi Shan ${ }^{1,2} \cdot$ Sudipa Sarkar ${ }^{1,3} \cdot$ Seth S. Martin ${ }^{1,4}$ (I)
}

Received: 25 January 2019 / Accepted: 13 March 2019 / Published online: 8 April 2019

(C) Springer-Verlag GmbH Germany, part of Springer Nature 2019

\begin{abstract}
Diabetes mellitus is a disease that can be difficult to manage and requires high levels of health literacy and numeracy, selfmonitoring and frequent contact with clinicians. If not optimally controlled, diabetes can lead to kidney failure, blindness and cardiovascular complications, which, in turn, contribute to increasing healthcare costs. Although not yet widely used, mobile health (mHealth) tools have enhanced diabetes management and prevention and are likely to play an increasing role with the growth of smartphone ownership and medical device innovations. Recent mHealth interventions targeting type 1 and type 2 diabetes are diverse in their goals and components, and include insulin management applications, wearable blood glucose meters, automated text messages, health diaries and virtual health coaching. In this paper, we review the modalities and components of various impactful interventions for insulin management, diabetes education, self-management and prevention. More work is needed to investigate how individual demographic, socioeconomic, behavioural and clinical characteristics contribute to patient engagement and the efficacy of mHealth tools for diabetes.
\end{abstract}

Keywords Diabetes $\cdot$ Digital health $\cdot$ Glucose monitoring $\cdot \mathrm{HbA}_{1 \mathrm{c}} \cdot \mathrm{mHealth} \cdot$ Precision medicine $\cdot$ Review $\cdot$ Self-monitoring $\cdot$ Smartphones $\cdot$ Wearables

$\begin{array}{ll}\text { Abbreviations } \\ \text { CE } & \text { Conformité Européenne } \\ \text { DID } & \text { Diabetes Interactive Diary } \\ \text { FDA } & \text { Food and Drug Administration } \\ \text { FTA } & \text { Few Touch Application } \\ \text { mHealth } & \text { Mobile health } \\ \text { mDPP } & \text { Mobile Diabetes Prevention Program } \\ \text { MITI } & \text { Mobile Insulin Titration Intervention } \\ \text { rtCGM } & \text { Real-time continuous glucose monitoring }\end{array}$

Electronic supplementary material The online version of this article (https://doi.org/10.1007/s00125-019-4864-7) contains a slide of the figure for download, which is available to authorised users.

Seth S. Martin

smart100@jhmi.edu

1 Ciccarone Center for the Prevention of Cardiovascular Disease, Division of Cardiology, Department of Medicine, Johns Hopkins University School of Medicine, Baltimore, MD, USA

2 David Geffen School of Medicine at UCLA, Los Angeles, CA, USA

3 Division of Endocrinology, Department of Medicine, Johns Hopkins University School of Medicine, Baltimore, MD, USA

4 Johns Hopkins Hospital, 600 N. Wolfe St, Carnegie 591, Baltimore, MD 21287, USA
SMBG Self-monitored blood glucose

SMS Short message service

\section{Introduction}

The growing field of mobile health (mHealth) has been applied to numerous areas, including health promotion, behaviour change support and self-management of chronic diseases. The US Food and Drug Administration (FDA) defines mHealth as the delivery of health services and improvement of health outcomes via mobile and wireless devices. mHealth interventions often employ modalities such as short message service (SMS) text messaging, smartphone applications ('apps') and wearable technology. mHealth is a subset of digital health or electronic health (eHealth), which also includes health information technology, telemedicine and personalised medicine [1]. Digital platforms can be adapted to changing medical guidelines and translated across different conditions. They can also be quickly scaled to reach thousands of people and potentially increase access to healthcare. In 2018 , approximately $66 \%$ of the world's population 
owned a smartphone, including up to $80 \%$ in Western European countries and $77 \%$ in the USA [2, 3]. Consumer technology companies, such as Apple, Google and Fitbit, have entered the healthcare market, and thousands of health or fitness mobile apps are in the Apple App Store and Google Play, though only a small proportion have been approved by entities such as the FDA [4].

The management of diabetes mellitus is challenging for both patients and clinicians. To successfully selfmanage diet, exercise, medications and insulin doses, patients must have high levels of health literacy and numeracy. Clinicians often motivate behaviour change, interpret blood glucose trends and adjust medication doses within brief clinic visits, sometimes engaging with patients who may have a limited understanding of their condition or treatment plan.

mHealth is well-suited to diabetes management, as it can provide frequent contact with patients and timely dissemination of health information, facilitate glycaemic control and guide self-management. A 2011 metaanalysis of 1657 individuals with type 1 or type 2 diabetes using SMS messages to send self-monitored blood glucose (SMBG) values and receive self-management information revealed a $0.5 \%(5.5 \mathrm{mmol} / \mathrm{mol})$ decrease in $\mathrm{HbA}_{1 \mathrm{c}}$ over 6 months in mHealth intervention groups compared with control groups, with a greater effect size in patients with type 2 diabetes than in those with type 1 diabetes [5]. A 2017 meta-analysis of 13 studies on mobile apps for diabetes suggested overall efficacy in reducing $\mathrm{HbA}_{1 \mathrm{c}}$, with a mean $0.44 \%(4.8 \mathrm{mmol} / \mathrm{mol} ; 95 \% \mathrm{CI}$ $0.29 \%, 0.59 \%)$ decrease in intervention compared with control, as well as increased perception of self-care among mobile app users [6]. Nevertheless, of the numerous commercially available mobile apps for diabetes, only 14 have clinical outcomes published in peer-reviewed literature or with regulatory clearance from the FDA or the Conformité Européenne (CE) mark, according to a 2016 review [7].

Initial results demonstrate the potential value of mHealth in diabetes. The field is rapidly expanding and the components of existing evidence-based interventions are heterogeneous. In this narrative review, we explore aspects of mHealth interventions for the management of diabetes, highlighting the various components of recent interventions.

\section{Insulin management}

The technical and computational challenges of calculating insulin boluses, counting carbohydrates, and titrating insulin make mHealth interventions for type 1 diabetes and insulindependent type 2 diabetes particularly valuable (Table 1 ).
Studied in patients with type 1 diabetes, Diabeo (Voluntis) [8] and Diabetes Interactive Diary (DID, Meteda) $[9,10]$ both consist of a mobile app that incorporates SMBG recording and an insulin bolus calculator. The bolus calculators use algorithms that consider SMBG values, carbohydrate intake and physical activity, as well as clinician-set parameters for the insulin/carbohydrate ratio, correction factor and basal insulin dose. The systems also recommend changes to these parameters if postprandial or fasting SMBG levels are off target. These systems differ in their telemedicine components and efficacy. While Diabeo offers biweekly telephone consultations with healthcare professionals, DID sends recorded data to clinicians via SMS messaging, and recommended changes in treatment or behaviours can be texted back to the patient $[8,10]$. A three-arm RCT found a $0.91 \%$ $(9.9 \mathrm{mmol} / \mathrm{mol})$ greater $\mathrm{HbA}_{1 \mathrm{c}}$ reduction at 6 months in the Diabeo plus telemedicine arm compared with usual care $(p<0.001)$, with no difference in hypoglycaemia [8]. Diabeo is commercially available in Europe [7], and a larger-scale RCT evaluating Diabeo with telemedicine support is underway in France to confirm the prior results [11]. In contrast, DID has been evaluated in two multicentre RCTs $[9,10]$, both of which did not demonstrate efficacy in $\mathrm{HbA}_{1 \mathrm{c}}$ reduction. However, the DID group had an $86 \%$ lower incidence rate of moderatesevere hypoglycaemia episodes and improved quality of life scores [10]. DID is commercially available in Italy and has been given the CE mark in Europe [7].

The pilot randomised Mobile Insulin Titration Intervention (MITI) trial [12] aimed to streamline insulin glargine titration among low-income, ethnically diverse type 2 diabetes patients via text messaging. Patients texted SMBG values, and a nurse provided algorithm-based insulin glargine titration advice via weekly phone calls. A higher proportion of patients in the intervention arm reached an optimal insulin glargine dose within the 12 week study ( $88 \%$ in the MITI arm, $37 \%$ in the control arm, $p<0.001)$, reducing frequent clinic visits for titration [12]. However, cost savings for the patient are offset by the need for uncompensated clinician intervention, as telemedicine reimbursements in the USA are currently limited. Generalisability is also limited, as MITI was designed for a specific patient population to integrate with a single clinic workflow; nevertheless, the simplicity and relative ease of set-up suggest that a texting intervention to meet a specific need is impactful and can be adapted for other uses.

\section{Connected and wearable blood glucose meters}

Many mobile apps incorporate connected blood glucose meters and are often developed by the device manufacturers. 


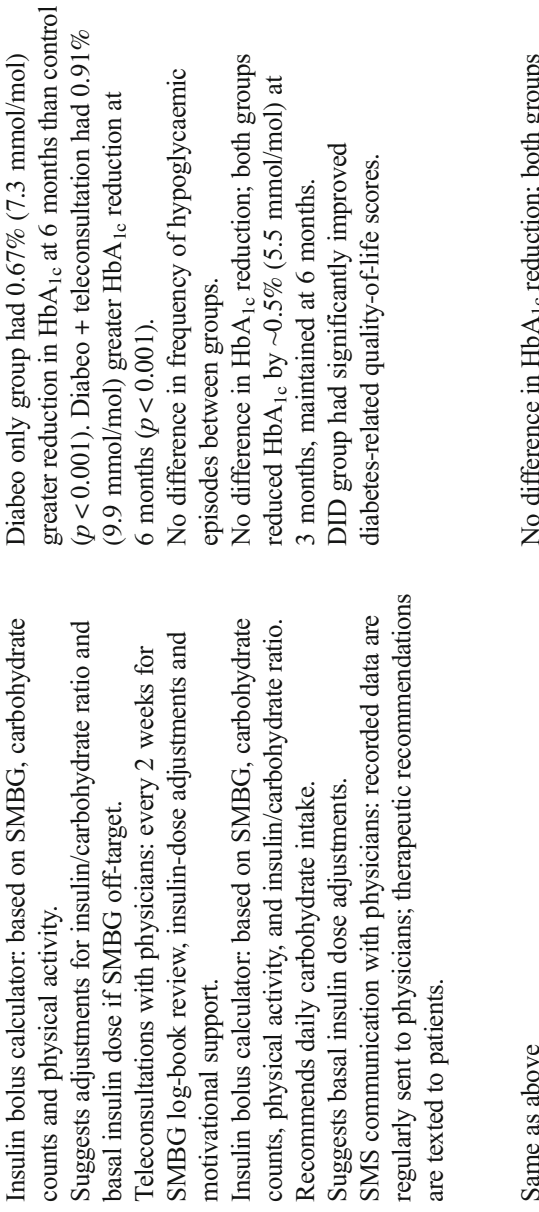

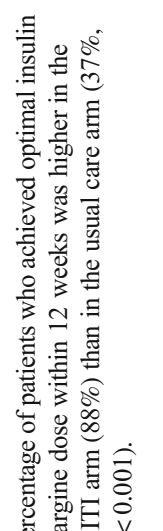

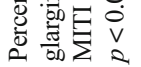

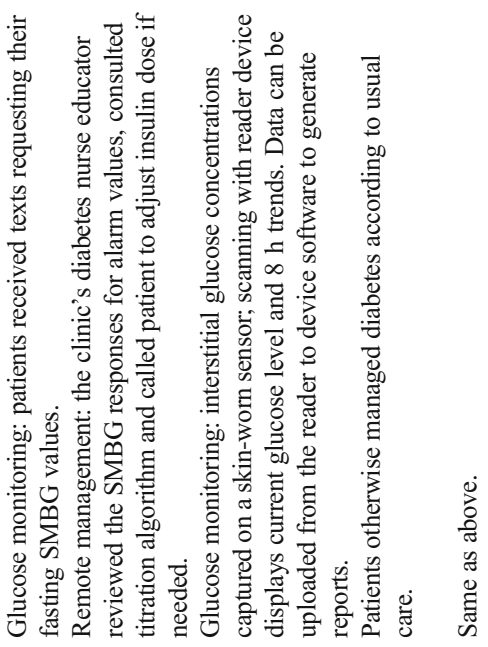

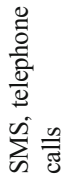

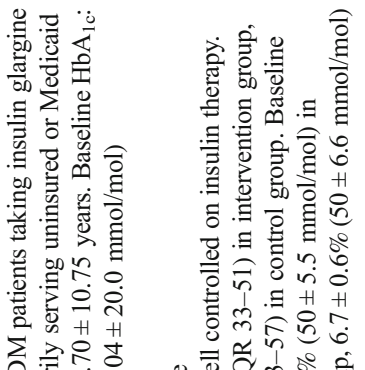

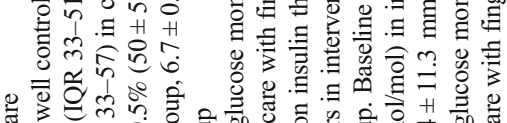

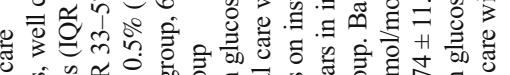

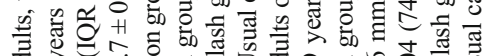

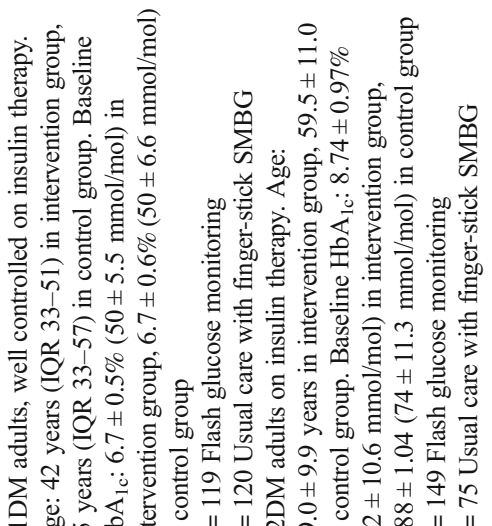

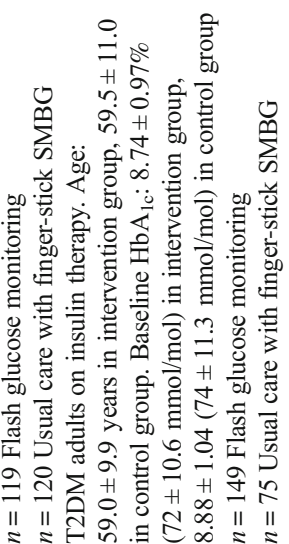

苞 $\overline{0}$

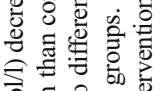
论

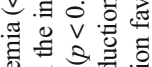
政 on 쳥녀 苗言言

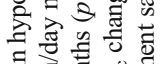

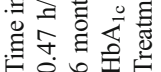

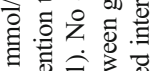

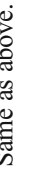

苋 政

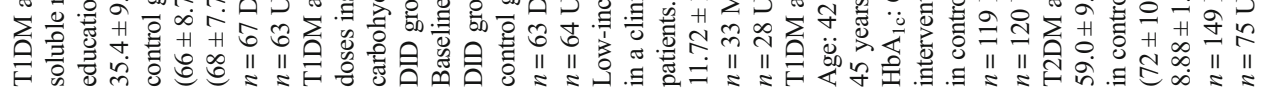

$\infty \&$

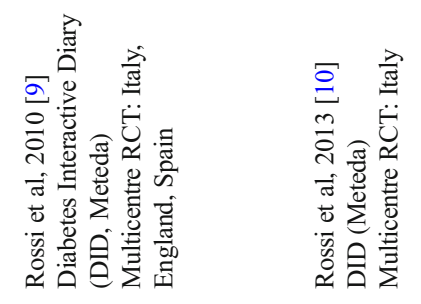

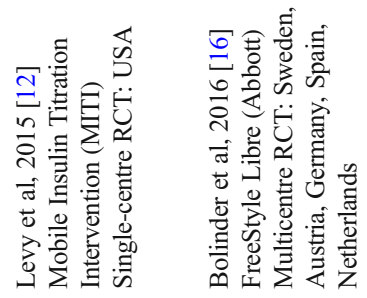

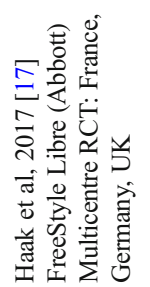


For example, the commercially available Accu-Chek Connect app (Roche) receives SMBG results from the Accu-Chek Connect blood glucose meter and includes an insulin bolus calculator and photographic food diary to aid in carbohydrate counting [7]. The ease of data flow from connected blood glucose meters allow glucose patterns to be efficiently presented to patients for self-management and to clinicians for treatment adjustments.

The inconvenience and pain of obtaining finger-stick blood samples from traditional blood glucose meters can contribute to poor adherence to self-monitoring and is one barrier to optimal glucose control. Real-time continuous glucose monitoring (rtCGM) and intermittently scanned flash glucose monitoring are increasingly used as adjuncts and alternatives to finger-stick glucose tests for making diabetes treatment decisions. Both use a wearable sensor that measures interstitial fluid glucose concentrations, which are transmitted to a reader device. Although rtCGM has been available for some time, flash glucose monitoring is a newer alternative to SMBG that can be used without calibration with a finger-stick test [13]. The FreeStyle Libre flash glucose monitor (Abbott) obtained FDA approval in 2017 and the CE mark in $2018[14,15]$ and is now available by prescription. The factory-calibrated disk-like sensor is worn on the upper arm for up to 14 days. By passing a reader device over the sensor, the patient can obtain real-time glucose levels and trends, and hypo- and hyperglycaemia alarms are available in some countries [15]. Large multicentre RCTs of the FreeStyle Libre sensor vs finger-stick SMBG demonstrated lower time in hypoglycaemia in type 1 diabetes patients [16] and in type 2 diabetes patients on insulin therapy [17]. Although studies have not shown decreased 6 month $\mathrm{HbA}_{1 \mathrm{c}}$ levels, the device promoted increased monitoring frequency (mean 8-15 scans per day with FreeStyle Libre vs $<1$ SMBG per day), with higher treatment satisfaction $[16,17]$.

Flash glucose monitoring and rtCGM are useful when higher frequency data are needed, for example, to generate a remotely viewed glucose log after making therapy adjustments, so clinicians can see if the changes were effective. Although these devices are increasingly common, the high costs (depending on insurance coverage) prohibit more widespread use. Despite concerns regarding the accuracy of interstitial fluid measurements, particularly at low glucose values and with equilibration delay between vascular and interstitial compartments, these methods have generally good concordance with SMBG [18]. The increased adherence to self-monitoring and higher patient satisfaction with flash glucose monitoring highlight the importance of the user experience in promoting self-management of diabetes.
Manufacturers such as Dexcom and Abbott have developed mobile apps supporting the rtCGM and flash glucose monitoring devices to enhance monitoring of glucose data. For example, the LibreLink (Abbott) mobile app allows a smartphone to replace the reader device, using near field communication to scan the sensor and read glucose data, displaying ambulatory glucose profile and estimated $\mathrm{HbA}_{1 \mathrm{c}}$ [13]. Additionally, carers of diabetic patients can use an app on their own smartphones to remotely monitor patients' glucose levels [13]. However, RCTs have focused on evaluating the safety and accuracy of the flash glucose monitoring devices exclusively, and studies describing the real-world use of the supporting mobile apps are lacking.

\section{Diabetes education, self-management and lifestyle modifications}

Clinicians often direct patients to attend in-person diabetes self-management classes, which may be burdensome, and this may be partly responsible for the low attendance rates [19]. Thus, mHealth interventions to support selfmanagement and diabetes education can potentially expand care delivery. Unlike interventions specifically designed for glucose monitoring or insulin dosing, mHealth interventions for education and self-management generally provide holistic content, are targeted towards patients with type 2 diabetes, and are informed by behavioural change theories, such as the Information-MotivationBehavioral Skills Model [20], social cognitive theory $[21,22]$, theory of planned behaviour [21] or motivational interviewing [23]. Patients are encouraged to self-monitor glucose, diet and exercise, and these data may be used to tailor feedback messages. Message content includes diabetes education, health promotion, motivational messages, reminders for medications and SMBG, or specific behavioural changes to implement, which are usually sent automatically according to an algorithm [20-22, 24-26]. The content of educational messages should be credible and evidence-based. Some interventions use expert-generated content; others are adapted from published national curriculums, such as the Diabetes Prevention Program [27], ADA's Diabetes Self-Management Education [25], or the National Diabetes Education Program [22] (Table 2).

Mobile applications with comprehensive features Several studies of smartphone applications have shown promising results in self-management of type 2 diabetes [20, 24, 28]. The BlueStar mobile diabetes coach (WellDoc) $[24,28]$ became the first type 2 diabetes app available on prescription [4] and a non-prescription version was approved by the FDA in 2017. BlueStar provides real-time automated educational and behavioural messages sent in response to 


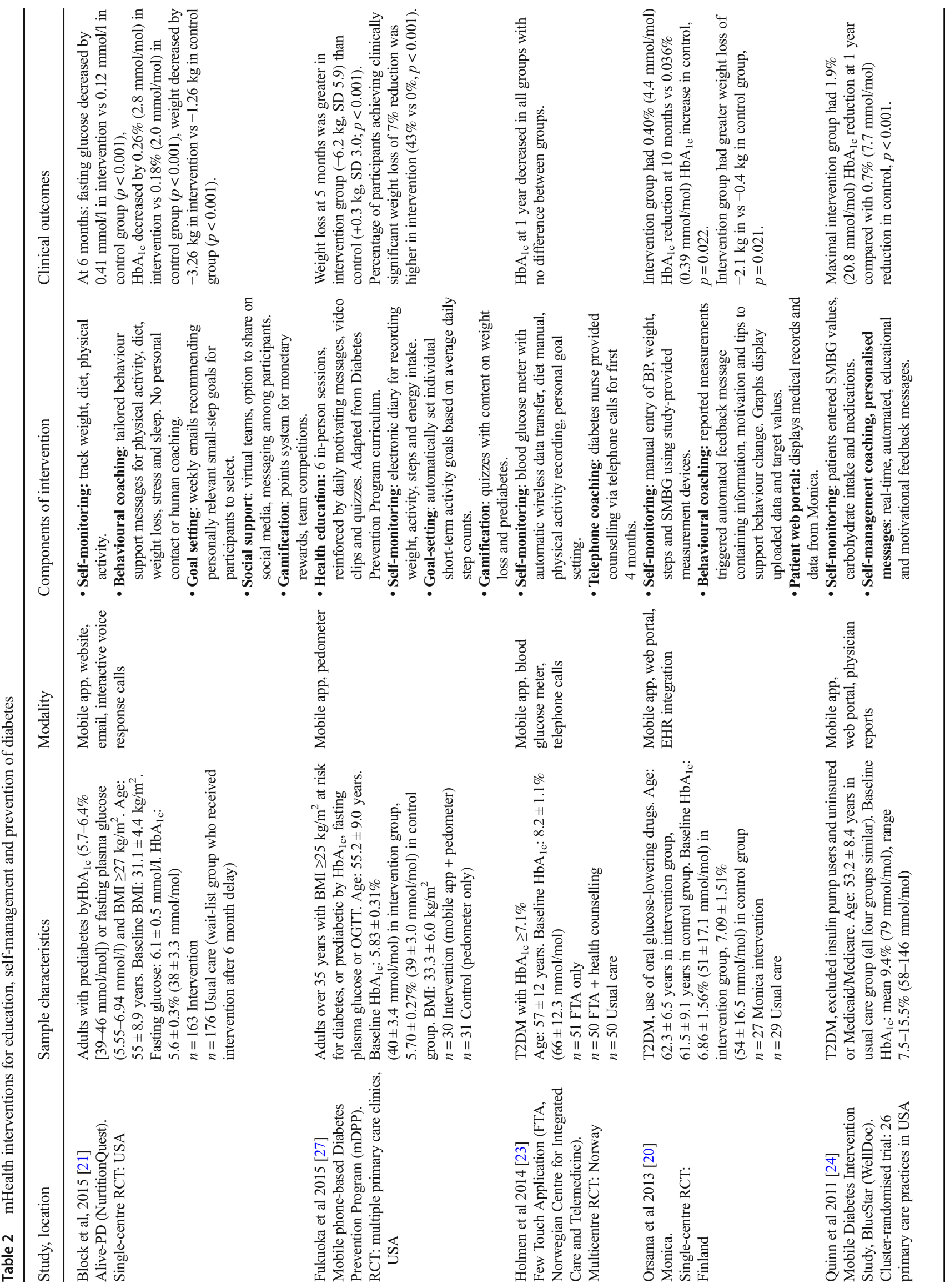




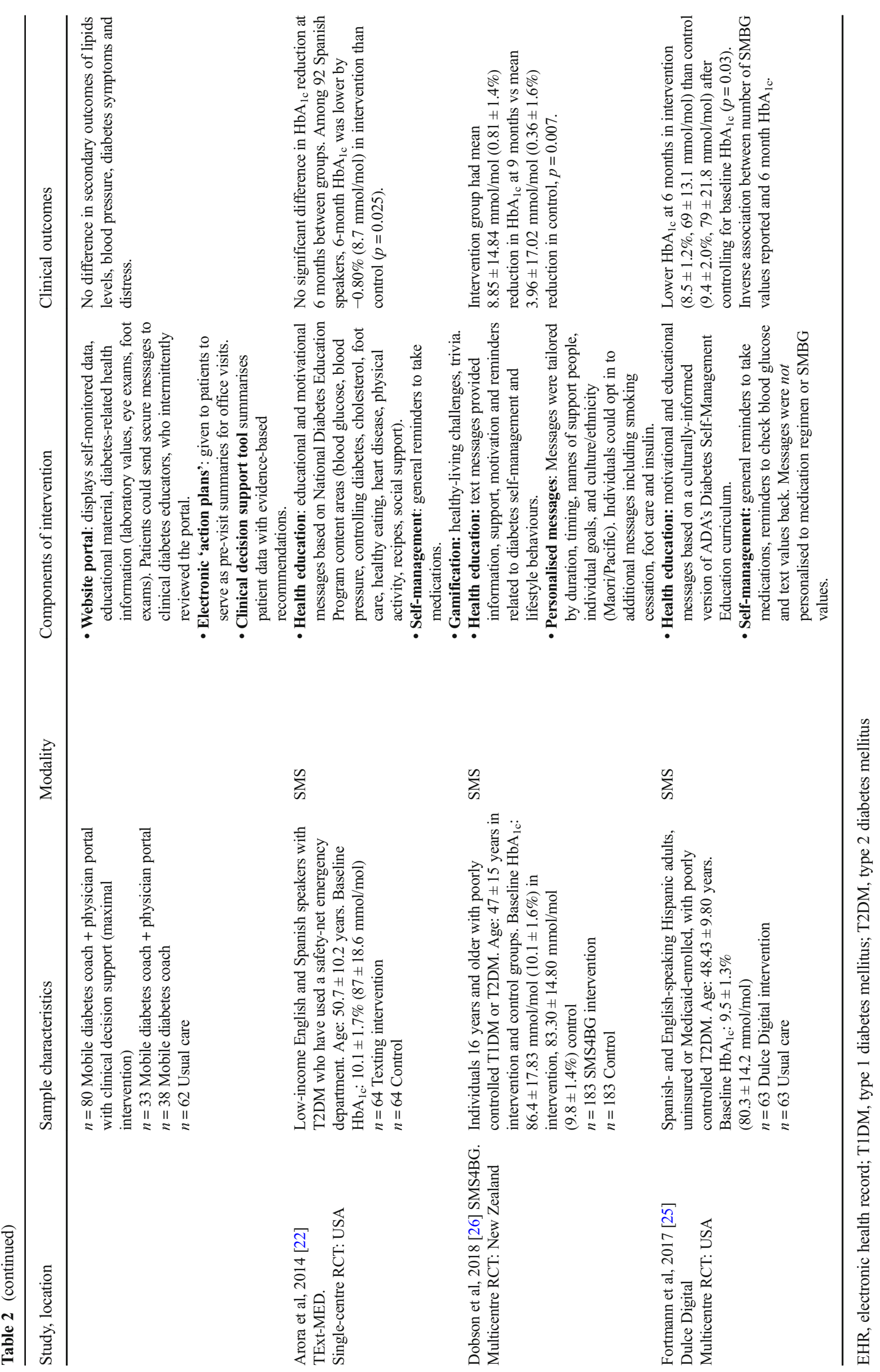


patient-reported data (including SMBG values, diabetes medications and lifestyle behaviours). In addition, patients securely message clinical diabetes educators though a webbased portal, and the patients' clinicians receive reports synthesising self-monitored data with treatment guidelines [24]. In a study from Finland, the Monica app sent automated patient-specific feedback messages in response to selfreported glucose, blood pressure, weight and steps. However, it did not provide any tools to support decision making by the clinician, despite having electronic health record integration and alerts to clinicians for concerning data [20]. Although RCTs for both interventions demonstrated reductions in $\mathrm{HbA}_{1 \mathrm{c}}$, BlueStar had a larger study population, involved multiple intervention arms and resulted in a larger effect $(1.2 \%$ [13.1 $\mathrm{mmol} / \mathrm{mol}]$ difference in $\mathrm{HbA}_{1 \mathrm{c}}$ reduction between maximal intervention arm and control, $p<0.001$ ), while the Monica results were less clinically significant $(0.436 \%$ [ $4.8 \mathrm{mmol} / \mathrm{mol}]$ difference in $\mathrm{HbA}_{1 \mathrm{c}}$ reduction between intervention and control, $p=$ 0.022) [20, 24]. However, the BlueStar RCT excluded patients who were uninsured or had Medicaid/Medicare insurance [24], limiting its generalisability. The Few Touch Application (FTA, Norwegian Centre for Integrated Care and Telemedicine) [23], a similar intervention, did not demonstrate any improvement in $\mathrm{HbA}_{1 \mathrm{c}}$. Although FTA promoted self-monitoring through a food and exercise diary and a wirelessly connected blood glucose meter, it did not incorporate real-time feedback messages, opting instead for monthly telephone coaching by a diabetes nurse accessing patient-reported data [23].

In patients at risk for diabetes, the Alive-PD (NutritionQuest) [21] behavioural change intervention and the Mobile Diabetes Prevention Program (mDPP) [27] were two important studies demonstrating the use of mobile apps to promote lifestyle modifications. Alive-PD is fully automated and consists of a mobile app and website for tracking physical activity, diet, weight loss, stress and sleep, with weekly emails to set individually tailored goals [21]. Unlike Alive-PD, the mDPP included six in-person sessions to deliver the curriculum. The mobile app reinforced content from the in-person sessions and allowed diet and step count tracking [27]. Full automation is more sustainable and scalable, but some patients may be more motivated and engaged with in-person sessions. Both interventions demonstrated efficacy in reducing body weight. Participants who received the $\mathrm{mDPP}$ intervention lost a mean $6.5 \mathrm{~kg}$ more than those in the control group $(p<0.001)$, while the Alive-PD intervention resulted in a mean $2 \mathrm{~kg}$ greater weight loss than control $(p<0.001)[21,27]$. mDPP did not affect $\mathrm{HbA}_{1 \mathrm{c}}$, a secondary outcome, while in Alive$\mathrm{PD}$, the reduction of $0.26 \%(2.8 \mathrm{mmol} / \mathrm{mol})$ in the intervention group vs $0.18 \%(2.0 \mathrm{mmol} / \mathrm{mol})$ in the control group $(p<0.001)$ is of uncertain clinical significance.
SMS-based diabetes education Simpler SMS-based interventions for diabetes self-management have been studied, including the recent Self Management Support for Blood Glucose (SMS4BG) study [26]. In this study, patients with poorly controlled type 1 or type 2 diabetes who were randomised to the treatment arm received educational and motivational text messages on self-management and lifestyle modifications, which were tailored by timing, duration, names of support people, individual goals and culture/ethnicity, with optional messages on smoking cessation, foot care and insulin management [26]. This relatively large study found a $4.89 \mathrm{mmol} / \mathrm{mol}(0.45 \%)$ greater decrease in $\mathrm{HbA}_{1 \mathrm{c}}$ in participants who received the intervention vs those given usual care. Despite this relatively modest effect, the study demonstrated that technology as straightforward as text messaging can be tailored in a sophisticated way.

SMS interventions are more accessible than smartphone applications, as they only require a basic cell phone without cellular data or WiFi; thus, these types of interventions may be more feasible in underserved populations. TExT-MED [22] and Dulce Digital [25] are two important US studies conducted among low-income, uninsured or Medicaid-enrolled English and Spanish speakers with type 2 diabetes. Both studies used nonpersonalised SMS messages to deliver culturally informed educational and motivational content, with reminders to take medications and check blood sugar. Although TExT-MED found no significant difference in $\mathrm{HbA}_{1 \mathrm{c}}$ improvement for the group as a whole, they found a significant and larger effect of the intervention among the 92 Spanish speakers enrolled, while Dulce Digital did find a significantly lower $\mathrm{HbA}_{1 \mathrm{c}}$ in the intervention group compared with the control group [22, 25]. The results suggest that the potential to lower $\mathrm{HbA}_{1 \mathrm{c}}$ through mHealthdelivered diabetes education may be greater in underserved populations, who may have lower health literacy and access to care at baseline.

\section{Access to remote clinicians}

Diabeo, DID, BlueStar and MITI all provide patients access to a remote clinician, which may contribute to efficacy but limit scaling, reproducibility and sustainability. For example, the insulin bolus calculator Diabeo is paired with teleconsultations for review of glucose logs, insulin dose adjustments and motivational support [8], while DID sends patient-reported data to physicians every $1-3$ weeks and allows texting between patients and physicians [9, 10]. However, data from Diabeo and DID are presented to clinicians without decision support tools, which may slow workflow due to data overload. Alternatively, MITI 
relies on a nurse-managed insulin titration algorithm to adjust therapy [12], while BlueStar provides clinicians with summaries of glycaemic control, self-reported medication adherence, and lifestyle behaviours alongside relevant evidence-based guidelines in a clinical decision support tool [24]. Of note, the intervention arm with the BlueStar app plus clinical decision support produced a $1.9 \%(20.8 \mathrm{mmol} / \mathrm{mol})$ decrease in $\mathrm{HbA}_{1 \mathrm{c}}$ at 1 year [24]. In these studies, involving the patient's own clinicians for feedback, motivation and treatment adjustments may enhance efficacy. For interventions to be applicable in the real world, integration with clinicians from the start of development is crucial to ensure the mHealth tool does not impede the existing workflow, and collaborations with payers and healthcare systems are necessary to ensure proper reimbursements for clinicians providing remote intervention. Indeed, reimbursements for telemedicine and remote patient monitoring have advanced but are still limited in scope in both France [29], where Diabeo was studied, and the USA, where MITI was studied.

\section{Patient engagement}

Patient engagement with technology, educational content and self-care behaviours influence outcomes of mHealth interventions. Thus, it is important to tailor the intervention in a patientcentred way and to evaluate user satisfaction. For example, although the DID intervention did not improve $\mathrm{HbA}_{1 \mathrm{c}}$, patients who used the app reported improved quality of life, including decreased 'fear of hypoglycaemia,' and improved 'social relations' [10]. In a satisfaction survey, $93.6 \%$ of patients who received the TExT-MED intervention enjoyed the programme and believed it was a good way to learn about diabetes [22].

Going beyond questionnaires, Quinn and colleagues performed a mixed-methods analysis of the messages that 107 BlueStar users sent to clinical diabetes educators via a web portal [30]. Patients who sent any messages during the 1 year study were significantly older and more likely to be white than those who did not send any messages. Most patients who never sent messages had a high school or lower level of education, perhaps indicating lower health literacy. Patient engagement was highest for more 'medical' topics, such as glucose monitoring and medications, and lower for 'lifestyle' topics, such as physical activity and healthy coping. Sending messages on any topic was associated with a $0.75 \%$ $(8.2 \mathrm{mmol} / \mathrm{mol} ; 95 \%$ CI $0.01 \%, 1.08 \%)$ lower $\mathrm{HbA}_{1 \mathrm{c}}$ compared with sending no messages [30]. Similarly, the Dulce Digital study found that the number of SMBG values sent by patients significantly correlated with lower 6 month $\mathrm{HbA}_{1 \mathrm{c}}$ values [25]. These results underscore the effect of patient engagement on outcomes and suggest that demographics and health literacy should be considered when designing interventions. However, contrary to the common belief that age is a barrier to mHealth adoption, older age does not necessarily impede engagement with technology [23, 30].

Some features designed to promote engagement include personalisation of messages, integration of social support and gamification, i.e. incorporating elements of game design into real-life concepts for non-gaming purposes, but there are no consistent methods of evaluating these features. Although personalised messaging may have been a component of success in BlueStar [24, 30], SMS4BG [26], Monica [20], and Alive-PD [21], results from the TExT-MED and Dulce Digital studies $[22,25]$ suggest that personalisation is not a requirement for efficacy or user satisfaction. The Alive-PD intervention promoted social support by creating virtual teams using a participant messaging system and the option to share content on social media [21]. Gamification methods include healthyliving challenges [22,31], team competitions, a points system with monetary rewards [21], trivia questions [22] and quizzes [21, 27]. Engagement may also be promoted with enhanced media, including video messages $[27,32]$ and voice recognition [33]. Despite the intuitive appeal of such features, more research is needed to specifically evaluate their impact on engagement outcomes, and to explore which features are most effective for which types of individuals.

\section{Future research needs}

The design of mHealth interventions should incorporate both patient and clinician feedback on lifestyle or workflow integration, respectively, as well as usability and content. Access to a remote provider, via teleconsultations or text messages, may enhance clinical efficacy and patient accountability, but more research is needed to establish the optimal balance between increasing patient-clinician interaction while preserving scalability. In addition, more research is needed to assess how clinicians currently incorporate patient-facing mHealth tools into their practice and the prevalence of mHealth use in various healthcare systems. In high-resource settings, CGMs are relatively common, but comprehensive mobile apps and SMS-based interventions may be less common, possibly reflecting individual and systemic barriers to more widespread mHealth adoption. Cost is one barrier to adoption and sustainability that should be characterised in economic analyses, both in terms of limited reimbursement for remote clinicians and in cost to the patient for devices.

The sample sizes in the RCTs presented vary widely, with the largest being SMS4BG, which enrolled 183 patients per arm [26]. Although these numbers may be sufficient to detect significant change in $\mathrm{HbA}_{1 \mathrm{c}}$, larger studies are needed to allow stratification to assess outcomes by individual characteristics or usage patterns, and to assess hard cardiovascular outcomes. 
Furthermore, mHealth is capable of delivering precision medicine, as it allows personalisation for different patients and collects frequent physiological and usage data to inform individualised analyses [34]. Future studies should investigate which patients would benefit most from the intervention, how they could be identified, and how their engagement could be enhanced. Of the studies discussed, follow-up duration ranged from 3 to 12 months, which is enough to detect initial changes in $\mathrm{HbA}_{1 \mathrm{c}}$, but studies with longer follow-up are needed to assess the sustainability of outcomes and retention of use.

mHealth has the potential to promote health equity by expanding access to care. Future studies should continue to evaluate interventions tailored for underserved populations, as MITI [12], TExT-MED [22], Dulce Digital [25], and SMS4BG [26] demonstrated that mHealth interventions are well accepted, feasible and efficacious in low-income ethnically diverse populations. Underserved populations have much to benefit from mHealth, as they have high rates of cell phone ownership, are more likely to depend on smartphones for internet access [35] and are disproportionally affected by diabetes and its complications [36].

Medication adherence is an important component of diabetes management that requires additional research.
Some studies, such as SMS4BG [26], TExT-MED [22], and BlueStar [24], included general reminders to take medications as part of diabetes coaching and education, but measuring medication adherence is a challenge. TExT-MED found an improvement in the selfreported Morisky Medication Adherence Scale with the SMS intervention [22], but there are obvious limitations to self-reported adherence. A few interventions have targeted medication adherence in type 2 diabetes [37-39], using methods such as electronic blisters, electronic pill dispensers and daily SMS reminders. However, results are mixed, and the studies relied on different methods of measuring adherence, such as pill counts or pharmacy refills.

Given the varying degrees of efficacy of the interventions presented, which are generally modest in scope, more work is needed to determine what factors would improve the efficacy of mHealth tools. While interventions addressing medication adherence and lifestyle modifications will remain relevant in a patient-managed disease, insulin management tools such as connected blood glucose meters may become less relevant as better closed-loop systems become readily accessible.

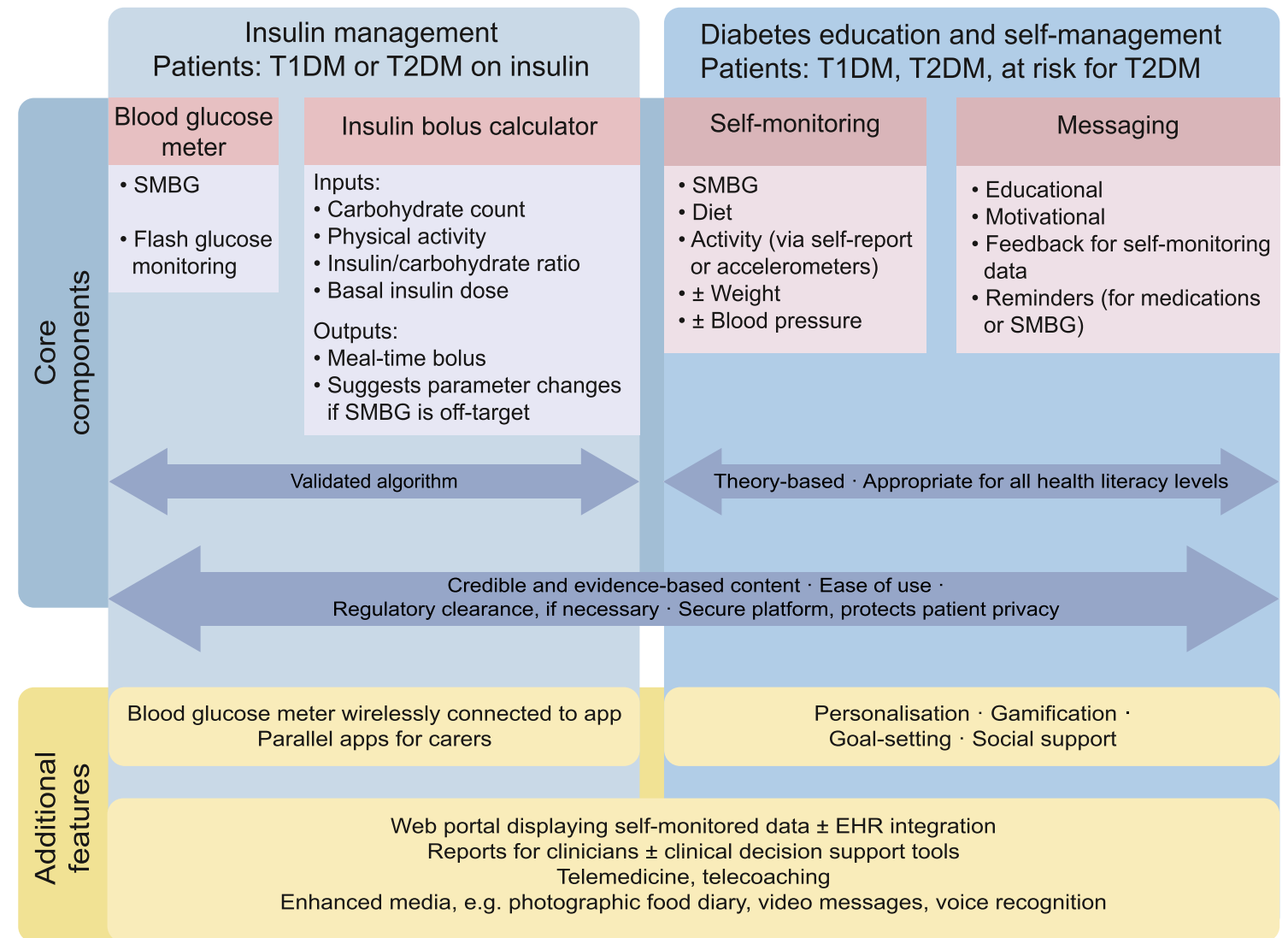

Fig. 1 Summary of key components and features of mHealth interventions for diabetes. EHR, electronic health record; T1DM, type 1 diabetes mellitus; T2DM, type 2 diabetes mellitus. This figure is available as a downloadable slide 


\section{Conclusion}

All the mHealth interventions presented (Table 1 and Table 2) were studied in RCTs, with most interventions (eight out of 13) demonstrating clinically and statistically significant efficacy, while five interventions had null results or achieved less than $0.5 \%$ ( $5.5 \mathrm{mmol} / \mathrm{mol})$ difference in $\mathrm{HbA}_{1 \mathrm{c}}$ reduction between intervention and control. These interventions vary from insulin bolus calculators and innovations in glucose monitoring to health education and lifestyle modifications. Their components include educational content, self-monitoring and automated messages providing motivation, education and feedback, as well as contact with a remote clinician through a telemedicine model (Fig. 1). Some features that may enhance patient engagement include personalised content, social support and gamification, but these have been inadequately studied. To allow more thoughtful personalisation of mHealth, more studies are needed to assess how individual factors, such as health literacy, culture, socioeconomic status, behaviours and treatment plan, impact patient engagement with mHealth tools and clinical outcomes.

\section{Summary}

- mHealth interventions for diabetes are heterogeneous and clinical effects are generally modest

- Barriers to adoption include cost, sustainability and integration with healthcare systems

- mHealth has particular potential to expand access to healthcare in underserved populations and to deliver precision medicine

- More work is needed to assess which features promote clinical efficacy and patient engagement

- Future work in diabetes should focus on medication adherence and lifestyle modifications

- Closed-loop systems may replace the need for insulin management mHealth tools

Funding This research received no specific grant from any funding agency in the public, commercial or not-for-profit sectors.

Duality of interest RS and SS report no conflicts of interest. SSM is a founder of and holds equity in Corrie Health, which intends to further develop the digital platform. This arrangement has been reviewed and approved by the Johns Hopkins University in accordance with its conflict of interest policies. SSM also reports receiving personal fees for serving on scientific advisory boards for Amgen, Sanofi, Regeneron, Quest Diagnostics, Akcea Therapeutics, Esperion and Novo Nordisk, as well as grants and research support from the PJ Schafer Cardiovascular Research Fund, the David and June Trone Family Foundation, American Heart Association, Aetna Foundation, Maryland Innovation Initiative, Nokia, iHealth, Google and Apple.
Contribution statement RS contributed to the literature search and writing the manuscript. SS and SSM contributed to writing the manuscript and revising for intellectual content. All authors approved the final version.

\section{References}

1. U.S. Food and Drug Administration (2018) Digital health. Available from www.fda.gov/medicaldevices/digitalhealth/. Accessed 9 Jan 2019

2. Zenith (2017) Smartphone penetration to reach $66 \%$ in 2018 . Available from www.zenithmedia.com/smartphone-penetrationreach-66-2018/. Accessed 9 Jan 2019

3. Pew Research Center (2018) Mobile fact sheet. Available from www.pewinternet.org/fact-sheet/mobile/. Accessed 9 Jan 2019

4. Neubeck L, Lowres N, Benjamin EJ et al (2015) The mobile revolution - using smartphone apps to prevent cardiovascular disease. Nat Rev Cardiol 12(6):350-360. https://doi.org/10.1038/ nrcardio.2015.34

5. Liang X, Wang Q, Yang X et al (2011) Effect of mobile phone intervention for diabetes on glycaemic control: a meta-analysis. Diabet Med 28(4):455-463. https://doi.org/10.1111/j.1464-5491. 2010.03180.x

6. Bonoto BC, de Araújo VE, Godói IP et al (2017) Efficacy of mobile apps to support the care of patients with diabetes mellitus: a systematic review and meta-analysis of randomized controlled trials. JMIR mHealth uHealth 5(3):e4. https://doi.org/10.2196/mhealth. 6309

7. Drincic A, Prahalad P, Greenwood D, Klonoff DC (2016) Evidence-based mobile medical applications in diabetes. Endocrinol Metab Clin N Am 45(4):943-965. https://doi.org/10. 1016/j.ecl.2016.06.001

8. Charpentier G, Benhamou P-Y, Dardari D et al (2011) The Diabeo software enabling individualized insulin dose adjustments combined with telemedicine support improves $\mathrm{HbA}_{1 \mathrm{c}}$ in poorly controlled type 1 diabetic patients: a 6-month, randomized, open-label, parallel-group, multicenter trial (TeleDiab 1 Study). Diabetes Care 34(3):533-539. https://doi.org/10.2337/dc10-1259

9. Rossi MCE, Nicolucci A, Di Bartolo P et al (2010) Diabetes Interactive Diary: a new telemedicine system enabling flexible diet and insulin therapy while improving quality of life: an open-label, international, multicenter, randomized study. Diabetes Care 33(1): 109-115. https://doi.org/10.2337/dc09-1327

10. Rossi MC, Nicolucci A, Lucisano G et al (2013) Impact of the "Diabetes Interactive Diary" telemedicine system on metabolic control, risk of hypoglycemia, and quality of life: a randomized clinical trial in type 1 diabetes. Diabetes Technol Ther 15(8):670 679. https://doi.org/10.1089/dia.2013.0021

11. Jeandidier N, Chaillous L, Franc S et al (2018) DIABEO app software and telemedicine versus usual follow-up in the treatment of diabetic patients: protocol for the TELESAGE randomized controlled trial. JMIR Res Protoc 7(4):e66. https://doi.org/10.2196/ resprot.9154

12. Levy N, Moynihan V, Nilo A et al (2015) The Mobile insulin titration intervention (MITI) for insulin adjustment in an urban, low-income population: randomized controlled trial. J Med Internet Res 17(7):e180. https://doi.org/10.2196/jmir.4716

13. Leelarathna L, Wilmot EG (2018) Flash forward: a review of flash glucose monitoring. Diabet Med 35(4):472-482. https://doi.org/10. 1111/dme.13584

14. Center for Devices and Radiological Health US FDA (2019) Premarket approval (PMA). Available from www.accessdata.fda. 
gov/scripts $/ \mathrm{cdrh} / \mathrm{cfdocs} / \mathrm{cfpma} / \mathrm{pma} . \mathrm{cfm}$ ?id=P160030. Accessed 9 Jan 2019

15. Abbott (2019) Abbott's Freestyle ${ }^{\circledR}$ Libre 2, with optional real-time alarms, secures CE mark. Available from http://abbott.mediaroom. com/2018-10-01-Abbott-s-FreeStyle-R-Libre-2-with-OptionalReal-Time-Alarms-Secures-CE-Mark-for-Use-in-Europe. Accessed 9 Jan 2019

16. Bolinder J, Antuna R, Geelhoed-Duijvestijn P et al (2016) Novel glucose-sensing technology and hypoglycaemia in type 1 diabetes: a multicentre, non-masked, randomised controlled trial. Lancet 388(10057):2254-2263. https://doi.org/10.1016/S0140-6736(16) 31535-5

17. Haak T, Hanaire H, Ajjan R et al (2017) Flash glucose-sensing technology as a replacement for blood glucose monitoring for the management of insulin-treated type 2 diabetes: a multicenter, openlabel randomized controlled trial. Diabetes Ther 8(1):55-73. https:// doi.org/10.1007/s13300-016-0223-6

18. Ajjan RA, Cummings MH, Jennings P et al (2018) Accuracy of flash glucose monitoring and continuous glucose monitoring technologies: implications for clinical practice. Diabetes Vasc Dis Res 15(3):175-184. https://doi.org/10.1177/1479164118756240

19. National Center for Chronic Disease Prevention and Health Promotion (2015) Emerging practices in diabetes prevention and control: Medicaid coverage for DSME. https://www.cdc.gov/ diabetes/pdfs/programs/stateandlocal/emerging_practices-dsme. pdf. Accessed 10 Jan 2019

20. Orsama A-L, Lähteenmäki J, Harno K et al (2013) Active assistance technology reduces glycosylated hemoglobin and weight in individuals with type 2 diabetes: results of a theory-based randomized trial. Diabetes Technol Ther 15(8):662-669. https://doi.org/10. 1089/dia.2013.0056

21. Block G, Azar KM, Romanelli RJ et al (2015) Diabetes prevention and weight loss with a fully automated behavioral intervention by email, web, and mobile phone: a randomized controlled trial among persons with prediabetes. J Med Internet Res 17(10):e240. https:// doi.org/10.2196/jmir.4897

22. Arora S, Peters AL, Burner E et al (2014) Trial to examine text message-based mHealth in emergency department patients with diabetes (TExT-MED): a randomized controlled trial. Ann Emerg Med 63(6):745-754.e6. https://doi.org/10.1016/j.annemergmed. 2013.10.012

23. Holmen H, Torbjørnsen A, Wahl AK et al (2014) A mobile health intervention for self-management and lifestyle change for persons with type 2 diabetes, part 2: one-year results from the Norwegian randomized controlled trial RENEWING HEALTH. JMIR mHealth uHealth 2(4):e57. https://doi.org/10.2196/mhealth.3882

24. Quinn CC, Shardell MD, Terrin ML et al (2011) Clusterrandomized trial of a mobile phone personalized behavioral intervention for blood glucose control. Diabetes Care 34(9):1934-1942. https://doi.org/10.2337/dc11-0366

25. Fortmann AL, Gallo LC, Garcia MI et al (2017) Dulce Digital: an mHealth SMS-based intervention improves glycemic control in Hispanics with type 2 diabetes. Diabetes Care 40(10):1349-1355. https://doi.org/10.2337/dc17-0230

26. Dobson R, Whittaker R, Jiang Y et al (2018) Effectiveness of text message based, diabetes self management support programme (SMS4BG): two arm, parallel randomised controlled trial. BMJ 361:k1959. https://doi.org/10.1136/bmj.k1959
27. Fukuoka Y, Gay CL, Joiner KL, Vittinghoff E (2015) A novel diabetes prevention intervention using a mobile app: a randomized controlled trial with overweight adults at risk. Am J Prev Med 49(2):223-237. https://doi.org/10.1016/J.AMEPRE.2015.01.003

28. Quinn CC, Clough SS, Minor JM et al (2008) WellDoc ${ }^{\mathrm{TM}}$ mobile diabetes management randomized controlled trial: change in clinical and behavioral outcomes and patient and physician satisfaction. Diabetes Technol Ther 10(3):160-168. https://doi.org/10.1089/dia. 2008.0283

29. Ohannessian R, Yaghobian S (2017) Financing telemedicine in France: a descriptive retrospective study. J Int Soc Telemed eHealth 5(GKR):e35

30. Quinn CC, Butler EC, Swasey KK et al (2018) Mobile diabetes intervention study of patient engagement and impact on blood glucose: mixed methods analysis. JMIR mHealth uHealth 6(2):e31. https://doi.org/10.2196/mhealth.9265

31. Burner E, Lam CN, DeRoss R et al (2018) Using mobile health to improve social support for low-income Latino patients with diabetes: a mixed-methods analysis of the feasibility trial of TExT-MED + FANS. Diabetes Technol Ther 20(1):39-48. https://doi.org/10. 1089/dia.2017.0198

32. Bell AM, Fonda SJ, Walker MS et al (2012) Mobile phone-based video messages for diabetes self-care support. J Diabetes Sci Technol 6(2):310-319. https://doi.org/10.1177/193229681200600214

33. Foltynski P, Ladyzynski P, Pankowska E, Mazurczak K (2018) Efficacy of automatic bolus calculator with automatic speech recognition in patients with type 1 diabetes: a randomized cross-over trial. J Diabetes 10(7):600-608. https://doi.org/10.1111/1753-0407. 12641

34. Antman EM, Loscalzo J (2016) Precision medicine in cardiology. Nat Rev Cardiol 13(10):591-602. https://doi.org/10.1038/nrcardio. 2016.101

35. Pew Research Center (2015) U.S. smartphone use in 2015 | Pew Research Center. Available from www.pewinternet.org/2015/04 01/us-smartphone-use-in-2015/. Accessed 9 Jan 2019

36. Beckles GL, Chou C-F (2016) Disparities in the prevalence of diagnosed diabetes - United States, 1999-2002 and 2011-2014. MMWR Morb Mortal Wkly Rep 65(45):1265-1269. https://doi. org/10.15585/mmwr.mm6545a4

37. Gatwood J, Balkrishnan R, Erickson SR et al (2016) The impact of tailored text messages on health beliefs and medication adherence in adults with diabetes: a randomized pilot study. Res Social Adm Pharm 12(1):130-140. https://doi.org/10.1016/j.sapharm.2015.04. 007

38. Vervloet M, van Dijk L, de Bakker DH et al (2014) Short- and longterm effects of real-time medication monitoring with short message service (SMS) reminders for missed doses on the refill adherence of people with type 2 diabetes: evidence from a randomized controlled trial. Diabet Med 31(7):821-828. https://doi.org/10.1111/dme. 12439

39. Brath H, Morak J, Kästenbauer T et al (2013) Mobile health (mHealth) based medication adherence measurement - a pilot trial using electronic blisters in diabetes patients. Br J Clin Pharmacol 76:47-55. https://doi.org/10.1111/bcp.12184

Publisher's note Springer Nature remains neutral with regard to jurisdictional claims in published maps and institutional affiliations. 